\title{
Research on the Key Technologies of a New CNC Turret Vertical Drilling and Milling Machine
}

\author{
Chun-Xian WANG ${ }^{1, a,{ }^{*}}$, Fan ZHANG $^{1, b}$, Yan-Fei ZHU ${ }^{1, c}$, Shuo-Shuo WEI ${ }^{1, d}$, \\ Sheng-Lai $\mathrm{HU}^{2, \mathrm{e}}$
}

${ }^{1}$ School of Mechanical Engineering, Xuancheng Campus of Hefei University of Technology, No.301, Xunhua Rd., Xuancheng, Anhui, China (242000)

${ }^{2}$ Xuancheng Jianlin Machinery Co., Ltd., No.4, Yingbin Rd., Xuancheng, Anhui, China (242057)

acxwang2004@126.com, b879179437@qq.com, ' ${ }^{\text {cforeveryanfei@163.com, }{ }^{d} 1072741332 @ q q . c o m, ~}$ jisky2006@yahoo.com

${ }^{*}$ Corresponding author

Keywords: Drilling and Milling Machine, Turret, Three-axis Feed, Tool Changing Trigger.

\begin{abstract}
The key technologies and design methods of a new $\mathrm{CNC}$ turret vertical drilling and milling machine, which functions between ordinary CNC drilling machine and vertical machining center, are studied in the paper to solve the problems such as high cost, low accuracy and efficiency and labor intensive in machining small-size, porous and multi-process parts with conventional machines. On the basis of analysis of features and requirements of the machine, a proper turret center is selected and overall structure of the machine determined. With three-dimensional modeling and assembly, the longitudinal and lateral feed transmission mechanism are designed to realize the machine's three-axis feed by combining the cross sliding table and the turret center's own Z-axis feed mechanism. The tool changing process of the original tool changing trigger mechanism and its problems are analyzed and a new tool changing trigger mechanism and its control are developed in detail, which can reduce tool changing time and increase machining efficiency effectively. The machine developed in the paper fills the market gaps between ordinary CNC drilling machine and vertical machining center, and practical application proves that it meets the machining requirements and has a good market prospect.
\end{abstract}

\section{Introduction}

There are various small-sizes, porous and multi-process parts in industries such as automotive, bearings, pneumatic components, painting equipments, air hydraulic machine, and so on. Conventionally these parts are processed with ordinary CNC drilling machines, vertical machining centers or other machines, which require multiple clamping during machining and leads to high labor intensity, long processing time, low production efficiency and high production cost. Therefore, there is an urgent need for a special CNC machine which could complete the drilling, reaming, reaming, countersinking, tapping and plane milling and other processes with one-time clamping for those small-size, porous and multi-process parts [1]. To this end, the key technologies of a new CNC turret vertical drilling and milling machine are studied and its overall structure, three-axis feed transmission mechanism and a new tool changing trigger mechanism designed in more detail. The machine fills the gap between ordinary CNC drilling machine and vertical machining center and is suitable for machining small size, porous and multi-process parts such as cylinder block and valve body of automobile hydraulic brake system, and solve the problems with conventional machines effectively [2].

\section{Overall Design of the Machine}

\section{Functional Analysis of the Machine}

The new CNC turret vertical drilling and milling machine is mainly used for small-size and porous parts processing with main functions of drilling, tapping, reaming, countersinking and plane 
milling. It is especially suitable for plane milling and porous drilling of cylinder block and valve body of automobile hydraulic brake systems. According to the investigation and analysis of the practical requirement, the machine's maximum drilling diameter is $12 \mathrm{~mm}$ when the part is of steel and $16 \mathrm{~mm}$ when of iron, maximum tapping diameter is M12, and maximum drilling depth $150 \mathrm{~mm}$.

\section{Choice of the Turret Center and Design of Its Power Transmission}

The turret center is the most important part for the machine with multi-process capabilities. In order to reduce the cost and time span of development, a turret center in markets is chosen directly for simplifying the design and production of the machine. By comparison and analysis comprehensively, a particular model turret center is determined finally. The turret center has six spindles with a relatively compact structure (Fig. 1) and maximum drilling diameter $12 \mathrm{~mm}$ for steel and $16 \mathrm{~mm}$ for iron, maximum tapping diameter M12, maximum drilling depth $150 \mathrm{~mm}$ and maximum tool's diameter $20 \mathrm{~mm}$, and meets the needs of the machine to be developed. It could automatically rotate the turret to replace the spindle when a tool needs to be changed.

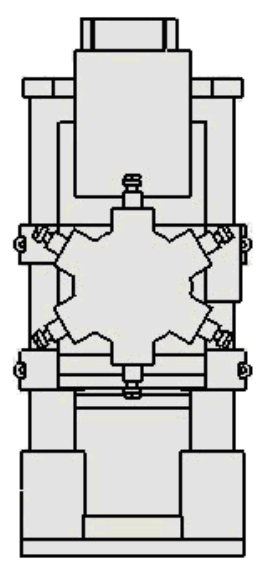

Fig.1 Exterior and structure of the turret center

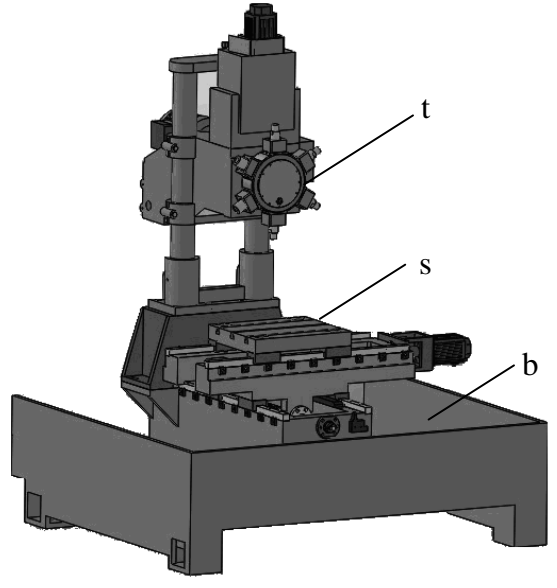

Fig. 2 Layout of the machine

In order to reduce the cost and the size of the machine, one motor is used for providing power for all spindles and the turret center itself simultaneously. The motor is referred to as spindle motor. The power transmission for spindles of the machine is as follows. With a synchronous belt, the spindle motor transmits its power to the turret center which transmits the power to the spindle for machining when the machine is in cutting status or to its internal mechanism for rotating the turret and replacing the spindle when in status of tool changing [3].

\section{Layout Design of the Machine}

Universal milling and drilling machines can be divided into vertical and horizontal structures with spindles set vertically for vertical structure and horizontally for horizontal structure. There are more tools for vertical drilling and milling machine so that the machine is more flexible and can be used for drilling, tapping, keyseat and plane milling, boring while a horizontal structured machine has fewer tools and can be used for keyseat and plane milling and cutting. Though a horizontal structured machine can provide some functions of a vertical structured machine with a special vertical milling head, it is poorer than a vertical structured one in functions and rigidity. Generally speaking, the general functions of a horizontal structured machine is more powerful while a vertical structured one is more productive. Add all this together with functions, workpiece clamping and chip removal during machining, the machine developed is vertically structured.

Fig. 2 is the 3D model of the machine by a 3D modeling software [4]. It can be seen that the machine mainly consists of three parts, which are the turret center, the sliding table and the base. The turret center integrates rotating tool magazines of six stations, and can rotate automatically to replace the spindle and tool for rapid tool changing $[5,6]$. The sliding table mainly consists of a longitudinal feed carriage and a lateral one, and can move longitudinally, laterally and vertically. Its 
outline dimension is $900 \mathrm{~mm}$ long and $420 \mathrm{~mm}$ wide, and multiple workpiece can be machined in one clamping. The upper surface of the base (Fig. 3) is used for installing the turret center and the sliding table while middle recessed part for bearing seats. The rest part of the upper surface is inclined for easy chip removal.

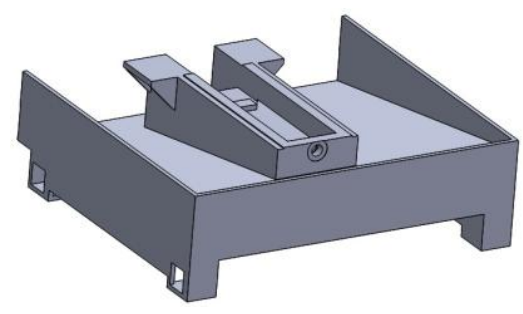

Fig. 3 The 3D model of the base

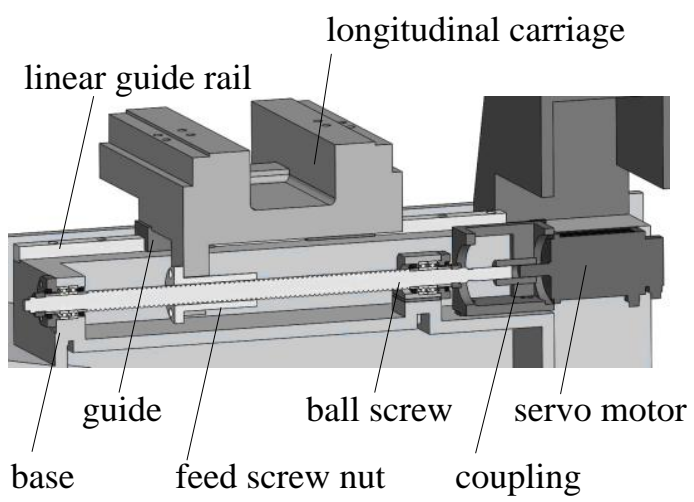

Fig. 4 The 3D model of longitudinal feed transmission mechanism

\section{Design of Three-axis Feed Transmission Mechanism}

As the turret center has its own Z-axis feed mechanism, it needs only to design a sliding table with longitudinal and lateral feed capabilities for realizing three-axis feeding.

\section{Design of Longitudinal Feed Transmission Mechanism}

After determination of the feed transmission scheme and calculation for the selection of ball screw, linear motion guide, servo motor and other parts, the longitudinal feed transmission mechanism is designed according to the shape and size of each part [7]. Fig. 4 is its 3D model. The linear guide rail is fixed on the base and the longitudinal carriage on the guide. The servo motor is connected to the ball screw with a coupling and transforms its rotation into linear movement of the longitudinal carriage by the feed screw nut.

\section{Design of Lateral Feed Transmission Mechanism}

The lateral feed transmission mechanism is designed similarly on the basis of longitudinal feed transmission mechanism and the structure and size of selected ball screw, linear motion guide, servo motor and other main parts (Fig.5). The main difference between the lateral and longitudinal feed transmissions is the moving distance and positions. The lateral feed transmission mechanism is installed on the longitudinal carriage while the longitudinal feed transmission mechanism on the base. So the lateral feed transmission mechanism can provide both longitudinal and lateral feed motions. The worktable is installed on the lateral feed transmission mechanism and has standard T-shape grooves and threaded holes on its upper surface for facilitating fixing of workpiece and fixtures.

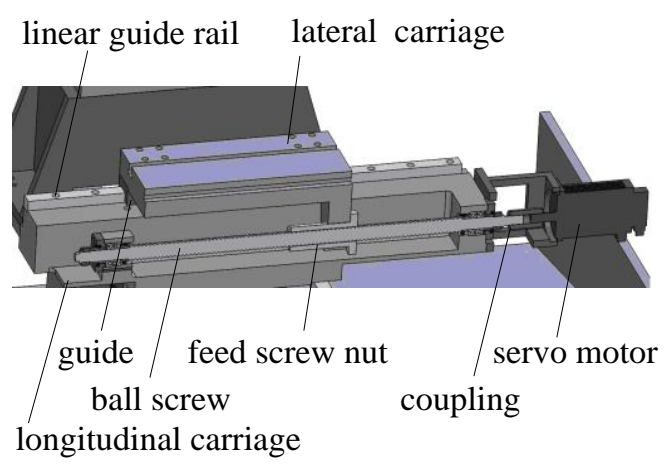

Fig.5 The 3D model of lateral feed transmission mechanism

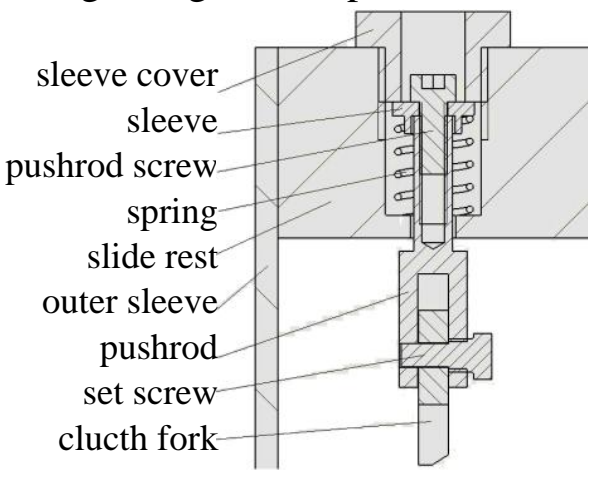

Fig. 6 The original tool changing trigger mechanism 


\section{Design of a New Tool Changing Trigger Mechanism}

\section{The Original Tool Changing Trigger Mechanism and Its Problem}

The original tool changing trigger mechanism of the turret center is shown in Fig. 6. It is installed on the slide rest. The pushrod screw is tightened in the threaded hole of pushrod while the sleeve is between the pushrod screw and the pushrod and the spring compressed between the sleeve and the slide rest. The tool changing trigger process is as follows. When the control system receives a tool changing instruction, it will start the Z-axis servo motor to drive the slide rest moving upwards. When the pushrod screw hits the pressing bar fixed on the machine body, the pressing bar will drive the pushrod screw moving downwards and make the sleeve compressing the spring and the pushrod moving downwards until the clutch fork acts to change the tool. After tool changing, the control system controls the servo motor to reverse and makes the slide rest moving downwards, and the pushrod screw breaks away from pressing bar and the sleeve return to its original position by the spring force (Fig. 7).
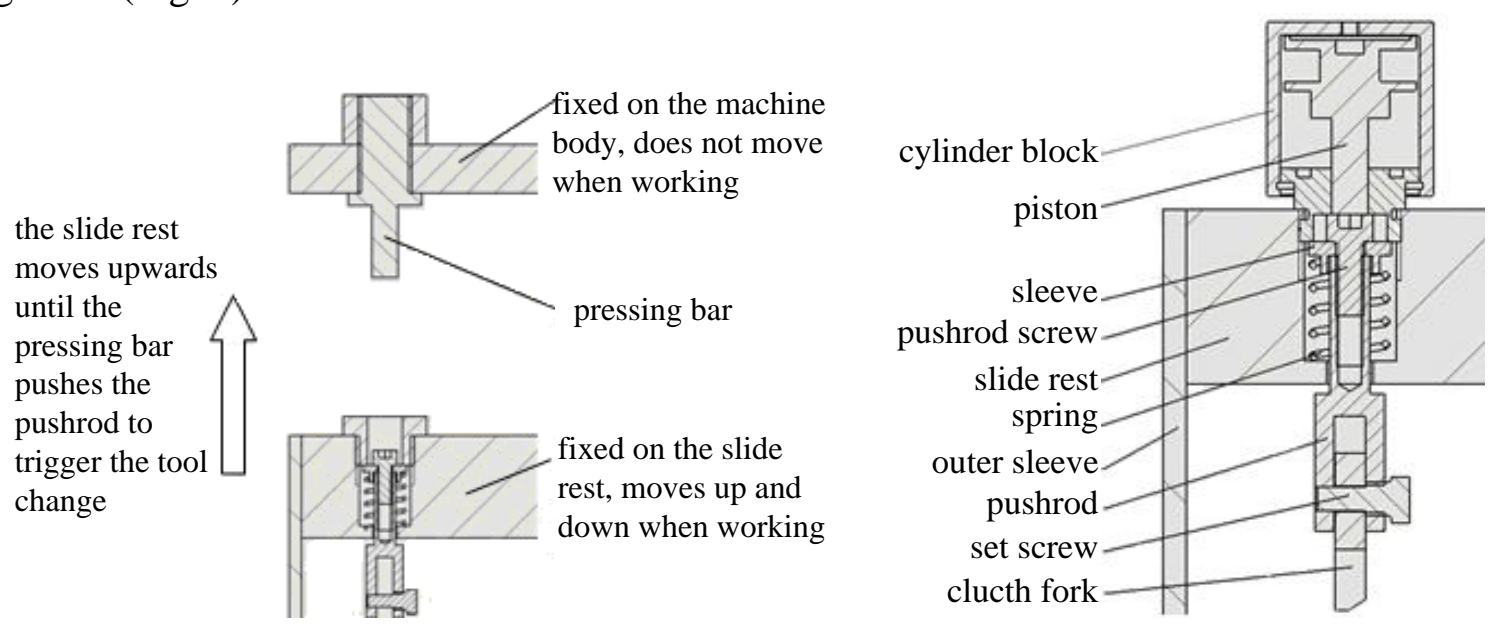

Fig. 7 Illustration of tool changing trigger Fig. 8 The new tool changing trigger mechanism

In each tool changing process, the slide rest must move upwards to the limit position for triggering the tool changing action and downwards for a certain distance to ensure an enough space for the pushrod back to its original position and avoid unwanted tool changing. Before tool changing action, a tool is generally in the machining status. When the tool needs to be changed, the slide rest has to move from the bottom to the top of the Z-axis guide rail to make the pressing bar hitting the pushrod screw. This up-and-down motion leads to longer tool changing time and low efficiency.

\section{Improvement of the Tool Changing Trigger Mechanism and Its Control}

A new tool changing trigger mechanism is designed to solve the problem of the original tool changing trigger mechanism (Fig. 8). As the central problem with the original one is the up-and-down motion of the slide rest during tool changing, an actuating device is chosen in the new mechanism to replace the pressing bar. The actuating device presses the pushrod screw directly and promptly in stead of the up-and-down motion of the slide rest during tool changing and thus the tool changing time is shortened. Because of the advantages of no pollution and low cost of air cylinder and small thrust for the pushrod to act, an air cylinder is chosen as the actuating device. A specific air cylinder is selected according to the parameters such as free length, elastic coefficient and outer diameter of the spring, the stroke of piston and thrust for the pushrod to act through kinematics and dynamic calculations [8].

In order to ensure that the new tool changing mechanism could be triggered smoothly and reliably, the air cylinder should be controlled robustly. The control flow of the cylinder and the tool changing is shown as Fig. 9. When the control system receives a tool changing command, it compares the current tool signal with the required tool signal at first. If the result is inconsistent, the 
control system sends a tool changing pulse for positive rotation to the PLC and makes the corresponding output points of PLC valid. The corresponding intermediate relay is energized to open the solenoid valve and compressed air enters the cylinder to compel the piston pushing the pushrod to trigger the tool changing. When the tool is changed, the control system detects and compares again the current tool signal and the desired tool signal. If they are inconsistent still, the control system will send another tool changing pulse for positive rotation until both signals are consistent. The duration for each action of the cylinder can be controlled by setting effective duration for corresponding output point in the PLC program. In the entire tool changing process, the tool changing is completely triggered by the air cylinder without the motion of slide rest except the motion for a safe distance from the workpiece, and the tool changing action is quick and easy [9, $10]$.

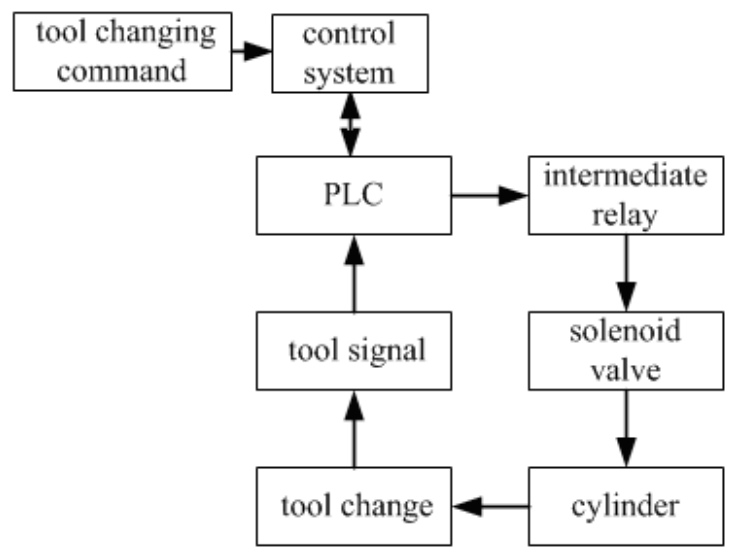

Fig. 9 Control flow of cylinder and tool changing

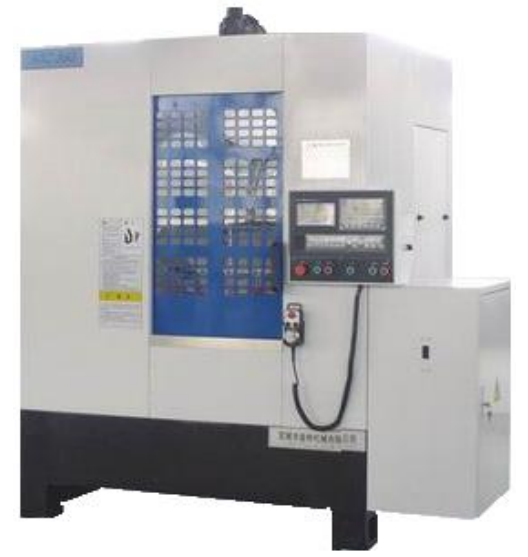

Fig. 10 Exterior of the machine

The actual test proves that the tool changing time between two adjacent tools is about 2.8 seconds with the original tool changing trigger mechanism and about 1.6 seconds with the new one, thus 1.2 seconds are saved. If the required tool is not adjacent to the current tool, the machine needs to trigger tool changing several times to switch to the desired tool and more time would be saved. There are six tools on the turret center and the machine needs to change tools frequently during machining. The new mechanism therefore can avoid a lot of waste of time and increase the machining efficiency significantly. As the improved mechanism only needs to add an air cylinder, an intermediate relay and a solenoid valve, there is almost no increase in manufacturing cost of the machine and the improvement is very practical.

\section{Conclusion}

A prototype machine has been manufactured successfully (Fig. 10). Practical applications prove that the machine meets the design requirements and can complete machining according to the required precision with automatic tool changing, high efficiency and low labor intensity. It fills the gap between the ordinary CNC drilling and vertical machining centers on the market by applying a turret center and is suitable for small size, porous and multi-process parts machining. It can be widely used in industries such as auto parts, bearings, pneumatic components, painting equipment, pneumatic and hydraulic parts, molds and sports equipment for drilling, reaming, countersinking, tapping, plane milling and other processes efficiently in one clamping with high precision by avoiding errors caused by multiple clamping.

\section{References}

[1] W.M. Yang, S.X. Zhao, P.F. Zheng and Z.X. Lou, Design of a kind of drilling machine for semicircle shaft, J. Advanced Materials Research, 10(2013) 328 331. 
[2] Y.L. Ling, Q.L. Ling and Z.W. Ning, Importance of CNC machining technology in the machinery manufacturing industry, J. China Science and Technology Information, 18 (2005) 76 76.

[3] M.Z. Zhang and G.Q. You, Drilling machine of CNC turret, CN. Patent CN2177552Y. (1994)

[4] X.W. Dang, Industrial design of mechanical product based on SolidWorks, J. Mechanical Research \& Application, 8(2010) 83 85.

[5] X.H. Su, Design of six-station turret tool machine, J. Manufacturing Technology \& Machine Tool, 31 (2010) 46 48.

[6] Z. Z. Huang, C. Liu and Z.H. Cheng, Design of automatic tool changer for machine center, J. Mechanical Engineering \& Automation, 21(2007) 124 125.

[7] Y.T. Bai, Feed drive system in digit control machine tool, J. Value Engineering, 6(2011), 7 8.

[8] Q.F. Wang, The design of micro cylinder based pneumatic, J. Hydraulics Pneumatics \& Seals, 4(2007) 47 48.

[9] T. Ji and C. Liu, Numerical control improvement on multi-situation tool turret, J. Machine Tool \& Hydraulics, 9(2006) 89 116.

[10] W. Liu, Automatic tool changer of NC machining center, J. Machine Tool \& Hydraulics, $5(2005) 58 \sim 58$. 